\title{
ASSESSING WATER-RELATED RISK FACTORS FOR BURULI ULCER: A CASE-CONTROL STUDY IN GHANA
}

\author{
HIROTSUGU AIGA, TAKAYUKI AMANO, SANDY CAIRNCROSS, JOSEPH A. DOMAKO, OFOSU-KWABI NANAS, AND \\ SUSAN COLEMAN \\ Emergency Needs Assessment Unit, United Nations World Food Programme, Rome, Italy; Department of Global Health, School of \\ Public Health and Health Services, Medical Center, The George Washington University, Washington, District of Columbia; \\ Department of Health Policy and Planning, School of International Health, Faculty of Medicine, The University of Tokyo, Tokyo, \\ Japan; Department of Neurosurgery, Yamaguchi University School of Medicine, Ube, Japan; Disease Control Vector Biology Unit, \\ London School of Hygiene and Tropical Medicine, London, United Kingdom; Ashanti Regional Health Administration, Ministry of \\ Health, Kumasi, Ghana
}

\begin{abstract}
To assess water-related risk factors of Buruli ulcer, a case-control study of 102 patients (51 cases and 51 controls) was undertaken by matching age group, sex, and bacille Calmette-Guérin (BCG) vaccination history in Ghana. The factors used here for matching have previously been implicated as factors of Buruli ulcer, an emerging infectious disease. This is the first study to delineate a set of previously suspected, water-related risk factors, in a case-control study matching for age group, sex, and BCG vaccination status. The results of both bivariate and multivariate analyses presented a significantly high odds ratio $(\mathrm{OR})$ only for swimming in rivers on a habitual basis $(\mathrm{OR}=18.00, P<0.01)$ among the major water-related risk factors. Use of water from rivers and ponds for drinking, cooking, bathing, and washing purposes were not significant risk factors. Our data suggest that swimming, or activities on riverbanks associated with it, is a risk factor.
\end{abstract}

\section{INTRODUCTION}

Buruli ulcer occurs in patchy foci in at least 27 countries in Africa, Asia, South America, and the western Pacific. The precise current distribution is not known ${ }^{1}$ and the incidence is probably underestimated. The majority of the reported cases are from west and central Africa: Benin, ${ }^{2}$ Côte d'Ivoire, ${ }^{3} \mathrm{Ga}-$ bon, ${ }^{4}$ Ghana, ${ }^{5,6}$ Liberia, ${ }^{7}$ Nigeria, ${ }^{8}$ Togo, ${ }^{9}$ Uganda, ${ }^{10}$ and Zaire. ${ }^{11}$ Since Bayley reported the first case of Buruli ulcer identified in Ghana in $1971,{ }^{12}$ awareness of the public health importance of the disease has been increasing. ${ }^{13-15}$

The disease was first described by Cook in Uganda in $1897^{16}$ and the etiologic agent was characterized by MacCallum and others in Australia. ${ }^{17}$ Since a great number of cases of the disease were identified in the Buruli area of Uganda, it is referred to as Buruli ulcer. Buruli ulcer is caused by $M y$ cobacterium ulcerans and often followed by extreme deformities and disabilities. ${ }^{18}$ Due to the ugly appearance of the deformities it leaves, Buruli ulcer is greatly feared and stigmatized in the endemic areas, and is often attributed to witchcraft and curses. ${ }^{19}$ Patients tend to be socially alienated. Since the treatment costs are extremely high, the appropriate medical care is not affordable for most local residents without financial support to either the health facilities or patients. ${ }^{20}$ At the least, treatment of this disease puts a considerable strain on health budgets.

Children less than 15 years of age are predominantly affected compared with adults. ${ }^{3,18,21}$ It is also reported that the prevalence in adult females is higher than in adult males, while there are no sex differences in the distribution of cases among children. ${ }^{1,22}$ An earlier study in $\mathrm{Uganda}^{23}$ reported that bacille Calmette-Guérin (BCG) might confer protection against the disease or delay the onset of symptoms. However, a more recent case-control study ${ }^{21}$ found that BCG vaccination did not protect against onset of the disease, though it shortened its duration.

Trauma to the skin followed by the introduction of $M$. ulcerans from a source of contamination is probably how infection begins. However, the mode of transmission has not yet been clearly demonstrated. A study in Benin reported the possibility of non-genetic familial person-to-person infection. ${ }^{2}$ Recently, a case of the disease following a human bite was reported. ${ }^{24,25}$ However, person-to-person transmission is clearly not a major route of transmission.

Epidemiologic studies suggest a connection with water because use of (or residence near) a river or pond has consistently been identified as a risk factor. ${ }^{3,8,10,26}$ Portaels and others suggested bites by or contact with insects inhabiting plant roots in swamps as a possible mechanism of M. ulcerans transmission. ${ }^{27}$ Marston and others, ${ }^{3}$ in one of only two published case-control studies of the disease, ${ }^{21}$ found that the wearing of long pants was protective. Nevertheless, the mechanism of transmission of the disease through water-related human activities is not yet identified. ${ }^{1}$ This study attempts to assess water-related risk factors in Ghana by controlling several other major possible risk factors.

\section{METHODS}

Study area and population. The site of the study, the Amansie West District in the Ashanti Region of Ghana, has the greatest number of reported cases of Buruli ulcer of the 18 districts in the region ${ }^{28}$ and the second greatest number in the country. ${ }^{15}$ The district is a typical tropical rain forest area of $1,320 \mathrm{~km}^{2}$ with an estimated total population of 130,000 in 135 communities. It is approximately $60 \mathrm{~km}$ southwest of Kumasi, the Ashanti regional capital. As one of the least developed districts in the country, infrastructure and socioeconomic conditions are poor. ${ }^{20}$

Within the district, the majority of Buruli ulcer-endemic communities are located in the south along the two rivers, the Offin and the Oda. Tontokrom is the most heavily affected community in the district with an estimated prevalence of $22 \% .{ }^{21}$ In addition to St. Martin's Catholic Hospital located in Agroyesum, which has been providing Buruli ulcer patients within and outside the district with curative services since $1993,{ }^{29}$ there are four government health centers and one private clinic in the district.

Study design. A case-control study was used to assess the water-related risk factors for Buruli ulcer. To estimate the 
level of risks specifically related to water such as type of water source and water-related behavior in daily life, the other major reported risk factors were matched between cases and controls or controlled against possible confounding.

Study population. The cases and controls were drawn from the catchment population of St. Martin's Catholic Hospital, including inpatients and outpatients seen in July and August 1999.

Case definition and confirmation. All the 51 patients with a diagnosis of Buruli ulcer were considered for inclusion as cases. These were confirmed by applying the Ziehl-Neelsen test $^{30}$ and polymerase chain reaction (PCR) methods ${ }^{31,32}$ to the tissue samples taken from their lesions for routine diagnosis purposes. The World Health Organization (WHO) recommends ${ }^{33}$ that the results of at least two such tests should be positive to confirm a diagnosis of Buruli ulcer. In the event, all cases were confirmed.

Laboratory methods. For the Ziehl-Neelsen test, specimens were prepared by flooding a smear on a slide with ZiehlNeelsen carbolfuchsin, heating for 3-5 minutes, rinsing with gentle stream of running water, counterstaining with methylene blue chloride (1 gram/liter), and air-drying. For the PCR, specimens were prepared by homogenizing tissue samples in a microfuge tube in $500 \mu \mathrm{L}$ of digestion buffer $(0.01 \mathrm{M}$ phosphate-buffered saline), centrifuging for five minutes, decanting the buffer, and resuspending the pellet in distilled water. Mycobacterium ulcerans DNA was isolated after heating the pellet at $95^{\circ} \mathrm{C}$. DNA amplification was performed in an automated thermal cycler.

Recurrent cases. The WHO criteria for recurrent cases were used: i.e., a patient with previous surgical treatment of Buruli ulcer who presents with another current lesion(s) at the same or different site(s) within one year from the end of the last treatment. ${ }^{1}$

Matching criteria. Considering the known epidemiology of the disease trends, individual matching was used as to age group and sex. Three age groups were arranged by using two cut-off points of 15 and 50 years old. ${ }^{1,21}$ Because of the possible association with BCG vaccination, frequency matching by vaccination status was used rather than individual matching because of the difficulty of identifying controls individually matched for all three factors: sex, age group, and vaccination status (Table 1). Since person-to-person transmission is not a major route of transmission, matching by familial infection (both genetic and non-genetic) was not undertaken, but it was included as a risk factor.

Sample size. The sample size was calculated so as to detect an association when the odds ratio (OR) was $\geq 3.5$ with $\alpha$

TABLE 1

Characteristics of Buruli ulcer cases used for matching*

\begin{tabular}{|c|c|c|c|c|c|}
\hline & $\frac{\text { Age category (years) }}{0-14}$ & \multicolumn{2}{|c|}{$\begin{array}{l}\text { Number } \\
\text { of cases }\end{array}$} & \multicolumn{2}{|c|}{ Percentage } \\
\hline Male & $\begin{array}{c}0-14 \\
15-49 \\
\geq 50\end{array}$ & $\begin{array}{r}15 \\
11 \\
4\end{array}$ & 30 & $\begin{array}{r}29.4 \\
21.6 \\
7.8\end{array}$ & 58.8 \\
\hline Female & $\begin{array}{c}0-14 \\
15-49 \\
\geq 50\end{array}$ & $\begin{array}{r}19 \\
1 \\
1\end{array}$ & 21 & $\begin{array}{r}37.3 \\
4.8 \\
4.8\end{array}$ & 41.2 \\
\hline $\mathrm{BCG}$ vaccination & $\begin{array}{l}\text { Yes } \\
\text { No }\end{array}$ & $\begin{array}{l}18 \\
33\end{array}$ & & $\begin{array}{l}35.3 \\
64.7\end{array}$ & \\
\hline
\end{tabular}

* For individual or frequency matching, the same number of controls were selected for each category.

$\mathrm{BCG}=$ bacille Calmette-Guérin. (error) $=0.05$ and $1-\beta$ (power) $=0.80$. Since the source of water among $30 \%$ of the rural residents in the country was either river, pond, or dugout, ${ }^{34}$ the proportion of controls exposed to water sources of those types was assumed to be 0.3 . This implied a requirement of 42 cases along with the same number of controls. ${ }^{35,36}$ Therefore, anticipating a $20 \%$ refusal rate, all 51 current inpatients and outpatients diagnosed as having Buruli ulcer at St. Martin's Catholic Hospital during the study period between July and August 1999 were selected as cases.

Selection of controls. Fifty-one controls were selected by systematic sampling based on treatment record from the inpatients and outpatients who received services at the hospital during the study period for health problems other than Buruli ulcer, such as malaria, respiratory infections, and diarrhea. The controls were selected from the same districts where cases resided. After selection of each case, subsequent treatment records were perused in chronological order until the first suitable control was found.

Data collection. To obtain data on types of water sources, hygienic behavior, swimming habit, and other household members' infection, structured interviews with cases and controls were conducted during home visits by using questionnaires. Interviews with current inpatients were undertaken at the hospital. When individuals were less than five years of age, their parents were interviewed. Akan, the local language in Ashanti Region, was used for interviews. The questionnaire was filled out by the interviewer during the interview. Repeated home visits were made until all the target interviewees were covered by the study. The full list of risk factor data collected for each case and control was as follows: age, sex, BCG status, types of water sources used, whether their drinking water was boiled and hands were regularly washed, habitual swimming in ponds and in rivers, any other cases in the household, involvement in agricultural work by the patient and by their parents/partner.

Statistical analysis. The WHO allows four types of water sources (piped water, well/borehole, river, and pond) in its standardized report forms for Buruli ulcer cases. ${ }^{1}$ Also, only these four types are accessible and used among the study population. Thus, these four types were used as the categories for water sources in this study. A fifth type, rainwater collection, was found to be extremely rare when the survey form was pretested. Data obtained through the interviews were field checked, entered into a computer, and then analyzed using SPSS for Windows version 11.0 (SPSS, Inc., Chicago, IL).

Statistical methods. First, a descriptive univariate analysis on cases was undertaken. Second, cases and controls were compared using bivariate analysis to give, for each potential risk factor variable, the OR, its $95 \%$ confidence interval and the corresponding $P$ value using the McNemar chi-square test. Third, logistic regression was undertaken for multivariate analysis, taking each patient's disease status (case or control) as a dependent variable. As the independent variables, we used the risk factors that showed significant associations $(P<0.05)$ in bivariate analyses. Logistic regression analysis was undertaken using forward stepwise variable entry. Variables were entered if they showed a significant association $(P<0.05)$ in the bivariate analysis and removed if not significant $(P>0.10)$ in the regression. 
Ethics. Informed consent to participate in the study was obtained verbally from both cases and controls after treatment and before the interview. In the case of children, consent was obtained from their parents. There were no refusals. Ethical approval was given by St. Martin's Catholic Hospital, following procedures laid down by the Ghana Ministry of Health.

\section{RESULTS}

Univariate analysis: characteristics of cases. All 51 cases treated at St. Martin's Catholic Hospital during the study period took part in the study (Table 1). Of these 51 cases, 34 (67\%) were children less than 15 years of age, but there were no cases less than two years of age. Among children (0-14 years of age), slightly more than half the cases were female (19 of $34,56 \%$ ), while most cases in both adult age groups were male (15 of $17,88 \%)$. This difference was statistically significant $\left(\chi^{2}=9.11, P=0.003\right)$. Of the 51 cases, $36(76 \%)$ were recognized as recurrent cases. Forty-two were from the Amansie West District and all the others were from adjacent districts. There were 102 active lesions in the 51 cases (mean of number of lesions per patient case $=2.0$ ). However, the unit of analysis in this study is the patient, not the lesion.

Bivariate analysis: ORs. The results of comparing cases and controls are shown in Table 2. Since multiple responses were allowed, the exposure groups in the table are not discrete. We therefore report the OR of Buruli ulcer for those reporting use of each type of source (or each type of behavior) compared with those not reporting it.

Seventeen cases $(33 \%)$ and nine controls (18\%) used more than one type of water source for drinking and cooking. Of all the cases and controls, only one case differentiated water sources according to purposes of water use. The household of this case used both well and river water for drinking and cooking, but only river water for bathing and washing. In Table 2, it is shown as using river water. All six cases using a piped supply were from the districts adjacent to the Amansie West District.

No controls used a piped water supply. We therefore combined piped supplies and wells/boreholes into a single cat- egory, piped supply or well/borehole, assuming the risks of these two types of water source were similar. This pooling process of two categories of water source is supported by the findings of an earlier study that Buruli ulcer is more frequent among households using water from a river or pond than in those using a piped supply, a borehole, or a well. ${ }^{26}$

Use of water from a river was associated with a significantly greater risk of disease $(\mathrm{OR}=2.38, P<0.05)$. However, use of water from a piped supply or well/borehole was not significantly protective $(\mathrm{OR}=0.55, P=0.332)$. Use of water from a pond was not significantly associated with disease, but the numbers of involved were small.

Of the four types of water-related behaviors we recorded, only swimming in rivers showed a significant positive association $(\mathrm{OR}=18.00, P<0.01)$. Only two controls swam on a habitual basis. Both were males from households where water from a well and a river was used. No cases or controls reported boiling water for drinking or swimming in ponds. And all cases and controls claimed to wash their hands before meals. Therefore, ORs could not be calculated for these three variables. Living with other household member(s) infected with Buruli ulcer appeared to be associated with an increased risk of disease in this study $(\mathrm{OR}=2.20)$, but the association was not significant. Only six (12\%) more cases than controls were exposed to this risk factor, suggesting that only $12 \%$ of the cases could be attributed to intra-household transmission. Being engaged in agricultural work did not present a significant risk factor, either for patients themselves or for their parents/partners.

Multivariate analysis: logistic regression. The risk factor variables that were significantly associated with Buruli ulcer $(P<0.05)$ were applied as the independent variables to logistic regression. These were swimming in rivers and domestic use of river water. As a result, the final model of the regression was composed of only one risk factor variable, swimming in rivers $(\mathrm{OR}=18.00, P<0.01)$. The constant in the logistic regression model was 0.105 . Naturally, the OR (18.00) is the same as that in the bivariate analysis.

We further analyzed the details on swimming in rivers. The number of cases who swam on a habitual basis was 19 and accounted for $37 \%$ of the total number of cases. Although no

TABLE 2

Results of bivariate analysis of risk factors for Buruli ulcer*

\begin{tabular}{|c|c|c|c|c|c|c|}
\hline & Risk factor variables & Cases & Controls & Odds ratio & $95 \%$ CI & $P$ \\
\hline \multirow[t]{4}{*}{ Water source $\dagger$} & Piped supply & 6 & 0 & 0.55 & $0.24-1.59$ & 0.332 \\
\hline & Well/borehole & 33 & 45 & 0.53 & & \\
\hline & River & 22 & 11 & 2.38 & $1.15-5.90$ & $0.049 \ddagger$ \\
\hline & Pond & 7 & 4 & 1.75 & $0.65-7.09$ & 0.549 \\
\hline \multirow[t]{4}{*}{ Water-related behavior } & $\begin{array}{l}\text { Boil water for drinking } \\
\text { (always twice or three times a day) }\end{array}$ & 0 & 0 & NA & NA & NA \\
\hline & $\begin{array}{l}\text { Wash hands before meal } \\
\text { (always twice or three times a day) }\end{array}$ & 51 & 51 & NA & NA & NA \\
\hline & Swimming in rivers§ & 19 & 2 & 18.00 & $3.77-362.13$ & $<0.001 \mathrm{~d}$ \\
\hline & Swimming in ponds & 0 & 0 & NA & NA & NA \\
\hline Familial cases & Other household member(s) infected & 12 & 6 & 2.20 & $0.90-7.25$ & 0.210 \\
\hline \multirow[t]{2}{*}{ Agricultural occupation } & Patient & 6 & 10 & 0.50 & $0.20-1.82$ & 0.381 \\
\hline & Patient's parent/partner & 38 & 27 & 2.38 & $1.15-5.90$ & 0.265 \\
\hline Total & & 51 & 51 & & & \\
\hline
\end{tabular}

$* \mathrm{CI}=$ confidence interval; $\mathrm{NA}=$ not applicable.

$\dagger$ Multiple responses allowed (more than one type of water source reported for 16 cases and 9 controls).

$\ddagger P<0.05$.

$\S$ Swimming on a habitat basis (once every two days or more).

II $P<0.01$. 
cases less than six years of age had a habit of swimming in rivers, those 6-14 years old who swam in rivers (10 cases) accounted for $67 \%$ of all cases among children. Of the 19 cases, 13 (68.4\%) were males and the others were females. Of these six female cases with the habit of swimming in rivers, five were between 6 and 11 years of age (Figure 1), and the other was 33 years old. Conversely, the male cases swimming habitually in rivers ranged in age from 10 to 59 years.

\section{DISCUSSION}

The predominance of children (2-14 years of age) among the cases is consistent with the finding of previous studies that children are the most affected by Buruli ulcer. ${ }^{1,3}$ Swimming in rivers on a habitual basis was the only significant risk factor among the eight variables studied through multivariate analysis. The strength of the association with swimming in rivers on a habitual basis $(\mathrm{OR}=18.00, P<0.01)$ was the greatest of the risk factor variables examined in this study and in previous studies. For instance, Marston and others reported ORs associated with residence in an encampment $(\mathrm{OR}=3.45, P<$ $0.01)$ and rice farming $(\mathrm{OR}=2.57, P<0.05){ }^{3}$ An earlier case-control study matching sex and age in the same district did not find significant risk factors related to water and thus did not discuss swimming in rivers. ${ }^{21}$ A study in Côte d'Ivoire assessed the various risk factors, but reported that swimming in rivers was not associated with a significant risk of Buruli ulcer. ${ }^{3}$ That study selected unmatched cases and controls, al-

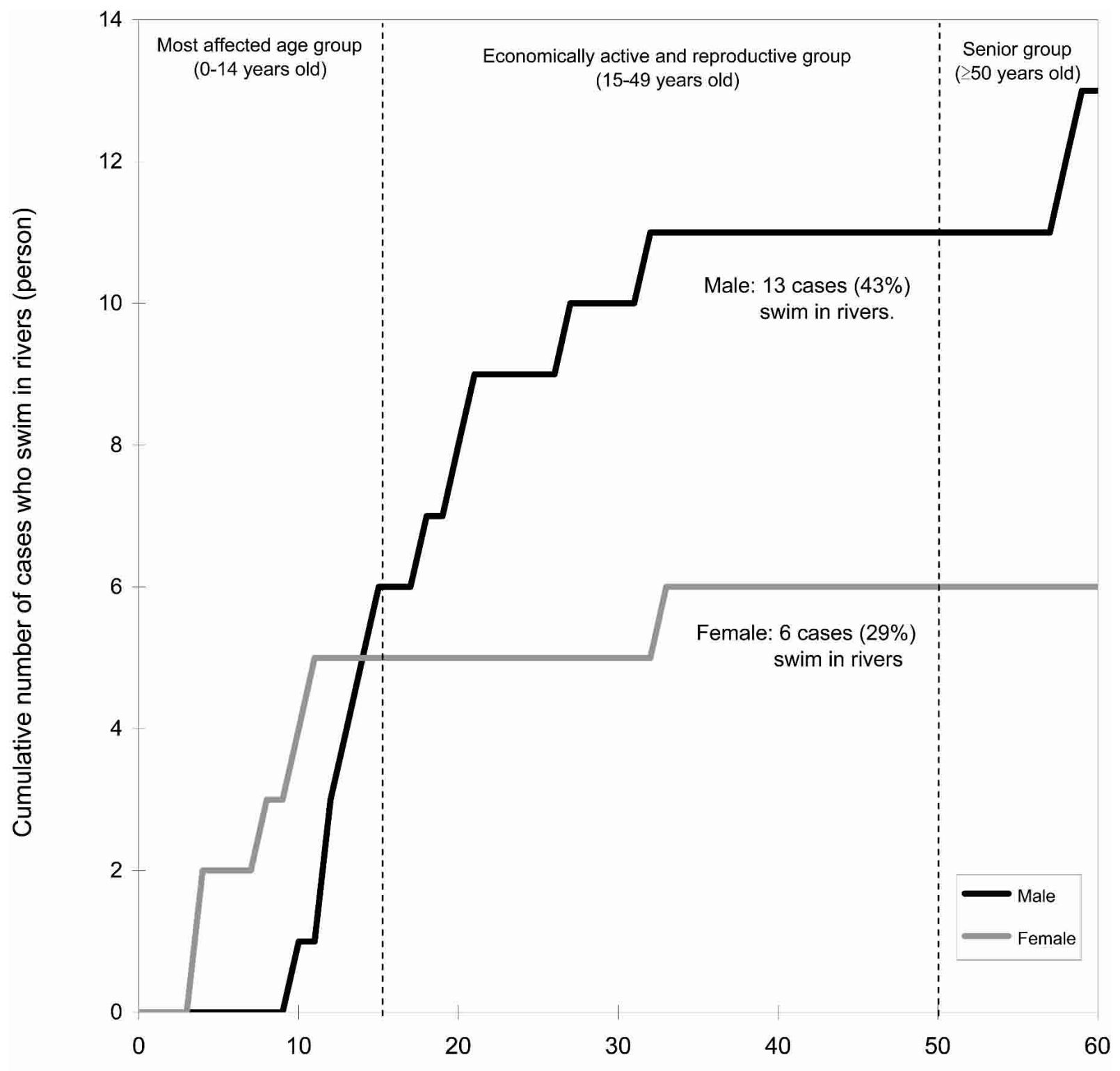

Age (year)

FIGURE 1. Cumulative number of cases who swam in rivers, by sex. 
though it included a multivariate analysis. Our matching for the well-known risk factors may have enabled our study to assess swimming in rivers as the critical water-related risk of Buruli ulcer with greater precision.

No case with the habit of swimming was found among children less than six years of age (Figure 1). Such younger children are probably too young to walk to the river by themselves. This supports the results of a study in Uganda. ${ }^{37}$ Swimming in rivers in rural areas is carried out generally for multiple purposes: 1) play or exercise, 2) fishing, 3) washing clothes, 4) fetching water, and 5) bathing. It is likely that the major purpose of children's swimming in rivers is play and exercise. Conversely, while only one $(17 \%)$ of the six female cases with a habit of swimming in rivers belonged to the economically active and reproductive age group (15-49 years of age), six (46\%) of the 13 male cases with that habit belonged to this age group. Those male adolescents and adults may swim in rivers not only for play and exercise but may also take the opportunity to fish, fetch water, and bathe. Swimming in rivers probably implies a variety of activities and their combinations.

Our sample of cases had the converse sex distribution from other studies. Other studies, including a nationwide survey of Ghana, have demonstrated a predominance of males among children $(<20$ years of age) with Buruli ulcer, but more females among adult cases ( $\geq 20$ years of age). ${ }^{15}$ This difference could be due to sampling error because our sample of cases is rather small.

Portaels and others hypothesized that bites of insects or contact with insect feces could be a mode of transmission. ${ }^{27}$ This could occur by contact with vegetation growing in stagnant water or poorly drained soil, as hypothesized for Uganda by Revill and Barker. ${ }^{37}$ Marston and others reported that wearing shoes or sandals could reduce the odds of disease by $67 \%(\mathrm{OR}=0.33)$, although it was not statistically significant. ${ }^{3}$ Unlike other activities in and near rivers (fishing, washing clothes, fetching water, and bathing), people who swim are unlikely to wear long pants or shoes. Taking these into account, the results of our study are consistent with the possibility that resting barefoot and scantily clad on riverbanks with abundant vegetation (where insects live) might bear the greatest part of the risk during the entire process of swimming activity. Unfortunately, our data cannot distinguish these activities from swimming itself.

We cannot exclude the possibility of transmission in the river itself. Rather, our results underline the need for further research. There are limitations in generalizing the findings of this study with small sample size at one hospital. However, we recruited all the current cases at St. Martin Catholic Hospital, where a larger number of Buruli ulcer patients are reported than at any other local health facility, which should have helped to reduce selection bias. Another limitation of our study is that we did not ascertain exactly how people swim in rivers and rest on riverbanks, what happens, and the detailed breakdown of time spent in the entire process of swimming. These factors need to be studied further.

The socioeconomic burden and loss through Buruli ulcer are contributing to a vicious cycle of poverty in the diseaseendemic areas. To break this vicious cycle, elucidation of the transmission route remains an urgent task.
Received December 18, 2003. Accepted for publication April 21, 2004.

Acknowledgments: We thank Dr. S. Etuaful and all the other staff of St. Martin's Catholic Hospital (Agroyesum, Ghana) and Dr. K. Aseidu (World Health Organization, Geneva, Switzerland) for their tremendous support in the data collection. We also thank Dr. D. Ofori-Adjei (Noguchi Memorial Institute for Medical Research, University of Ghana, Legon, Ghana) and Dr. A. Nakazawa (School of Medicine, Yamaguchi University) for their technical advice and support in the laboratory. This work is sincerely dedicated to those who live in Buruli ulcer-endemic areas worldwide.

Financial support: This work was supported by the Foundation for Advanced Studies on International Development, Japan.

Authors' addresses: Hirotsugu Aiga, Emergency Needs Assessment Unit, (FASID), United Nations World Food Programme, Via Cesare Giulio Viola, 68/70-Parco dé Medici, 00148 Rome, Italy, Telephone: 39-0-6513-2177, Fax: 39-6-6513-3080, E-mail: hirotsugu.aiga@wfp.org, Department of Global Health, School of Public Health and Health Services, Medical Center, The George Washington University $2175 \mathrm{~K}$ Street, NW, Suite 810, Washington, DC 20037, Telephone: 202-4160092, Fax: 202-496-0400, E-mail: ihphxa@gwumc.edu, and Department of Health Policy and Planning, School of International Health, Faculty of Medicine, The University of Tokyo 7-3-1 Hongo, Bunkyoku, Tokyo 113-0033, Japan, Telephone: 81-3-5841-3688, Fax: 81-35841-3637, E-mail: hiroaiga@f6.dion.ne.jp. Takayuki Amano, Department of Neurosurgery, Yamaguchi University Hospital, Yamaguchi University School of Medicine, 1-1-1 Minami-Kogushi, Ube, Yamaguchi 755-8505, Japan, Telephone: Phone: 81-836-22-2012, Email: fzf01304@nifty.ne.jp. Sandy Cairncross, Disease Control Vector Biology Unit, London School of Hygiene and Tropical Medicine, Keppel Street, London WC1E 7HT, United Kingdom, Telephone: 44-20-7927-2211, Fax 44-20-7636-7843, E-mail: sandy.cairncross@ lshtm.ac.uk. Joseph Adomako and Ofosu-Kwabi Nanas, Ashanti Regional Health Administration, Ministry of Health, PO Box 1908, Kumasi, Ashanti Region, Ghana, Telephone: 233-51-24809, E-mail: joseph_adomako@hotmail.com. Susan Coleman, Department of Global Health, School of Public Health and Health Services, Medical Center, The George Washington University, 2175 K Street, NW, Suite 810, Washington D.C. 20037, Telephone: 202-416-0406, Fax: 202-496-0400, E-mail: susancol@gwu.edu.

\section{REFERENCES}

1. World Health Organization, 2000. Asiedu K, Schepbier R, Raviglione M, eds. Buruli ulcer: Mycobacterium ulcerans infection. Geneva: World Health Organization, 12-13, 35-38, 37-38, 99-103.

2. Muelder K, Nourou A, 1990. Buruli ulcer in Benin. Lancet 336: $1109-1111$.

3. Marston BJ, Diallo MO, Horsburgh CR Jr, Diomande I, Saki MZ, Kanga JM, Patrice G, Lipman HB, Ostroff SM, Good RC, 1995. Emergence of Buruli ulcer disease in the Daloa region of Côte d'Ivore. Am J Trop Med Hyg. 52: 219-224.

4. Burchard GD, Bierther M, 1986. Buruli ulcer: clinical pathological study of 23 patients in Lambarene, Gabon. Trop Med Parasitol. 37: 1-8.

5. Addo HA, 1995. Mycobacterium ulcerans infection (Buruli ulcer) in Ga district of greater Accra region. Ghana Med J 29: 595602.

6. Amofah GK, Asamoah S, Afram-Gyening C, 1998. Effectiveness of excision of pre-ulcerative Buruli lesions in a rural district in Ghana. Trop Doct 28: 81-83.

7. Monson MH, Gibson DW, Connor DH, Kappes R, Hienz HA, 1984. Mycobacterium ulcerans in Liberia: a clinico-pathologic study in 6 patients with Buruli ulcer. Acta Trop 41: 165-172.

8. Oluwasanmi JO, Solankee TF, Olurin EO, Itayemi SO, Alabi GO, Lucas AO, 1976. Mycobacterium ulcerans (Buruli) skin ulceration in Nigeria. Am J Trop Med Hyg. 25: 122-128.

9. Meyers WM, Tignokpa N, Priuli GB, Portaels F, 1996. Mycobacterium ulcerans infections (Buruli ulcer): first reported patients in Togo. Br J Dermatol. 134: 1116-1121.

10. The Uganda Buruli Group, 1971. Epidemiology of Mycobacte- 
rium ulcerans infection (Buruli ulcer) at Kinyara, Uganda. Trans $R$ Soc Trop Med Hyg. 65: 763-775.

11. Meyers WM, Shelly WM, Connor DH, Meyers EK, 1974. Human Mycobacterium ulcerans infection developing at sites of trauma to skin. Am J Trop Med Hyg. 23: 919-923.

12. Bayley AC, 1971. Buruli ulcer in Ghana. BMJ 2: 401-402.

13. van der Werf TS, van der Graaf WT, Groothuis DG, Knell AJ, 1989. Mycobacterium ulcerans infection in Ashanti region, Ghana. Trans R Soc Trop Med Hyg. 83: 410-413.

14. Addy JH, 1995. The problem of Buruli ulcer in Ghana. Ghana Med J 29: 587-588.

15. Amofah GK, Bonsu F, Tetteh C, Okrah J, Asamoa K, Asiedu K, Addy J, 2002. Buruli ulcer in Ghana: results of a national case search. Emerg Infect Dis 8: 167-170.

16. Strickland GT, 2000. Hunter's Tropical Medicine and Emerging Infectious Diseases. Eighth edition. Philadelphia: W. B. Saunders Company, 524-525.

17. MacCallum P, Tolhurst JC, Buckle B, Sissons HA, 1948. A new mycobacterial infection in man. J Pathol Bacteriol 60: 93122.

18. Ellen DE, Stienstra Y, Teelken MA, Dijkstra PU, van der Graaf WT, van der Werf TS, 2003. Assessment of function limitations caused by Mycobacterium ulcerans: towards a Buruli ulcer functional limitation score. Trop Med Int Health 8: 90-96.

19. Stienstra Y, van der Graaf WT, Asamoa K, van der Werf TS, 2002. Belief and attitudes toward Buruli ulcer in Ghana. Am J Trop Med Hyg. 67: 207-213.

20. Asiedu K, Etuaful S, 1998. Socioeconomic implications of Buruli ulcer in Ghana: a three-year review. Am J Trop Med Hyg. 59: 1015-1022.

21. Amofah GK, Sagoe-Moses C, Adije-Acquah C, Frimpong EH, 1993. Epidemiology of Buruli ulcer in Amansie West district, Ghana. Trans $R$ Soc Trop Med Hyg. 87: 644-645.

22. World Health Organization, 2003. Facts about Buruli Ulcer. http://www.who.int/gtb-buruli/ (accessed May 2003).

23. Smith PG, Revill WD, Lukwago E, Rykushin YP, 1976. The protective effect of BCG against Mycobacterium ulcerans disease: a controlled trial in an endemic area of Uganda. Trans $R$ Soc Trop Med Hyg 70: 449-457.

24. Debacker M, Zinsou C, Aguiar J, Meyers W, Portaels F, 2002.
Mycobacterium ulcerans disease (Buruli ulcer) following human bite. Lancet 360: 1830.

25. Debacker M, Zinsou C, Aguiar J, Meyers W, Portaels F, 2003. First case of Mycobacterium ulcerans disease (Buruli ulcer) following a human bite. Clin Infect Dis 36: e67-e68.

26. Barker DJP, 1973. Epidemiology of Mycobacterium ulcerans infection. Trans R Soc Trop Med Hyg. 67: 43-47.

27. Portaels F, Elsen P, Guimaraes-Peres A, Fonteyne PA, Meyers WM, 1999. Insects in the transmission of Mycobacterium ulcerans infection (letter). Lancet 353: 986.

28. Amofah GK, 1995. Control and management of Buruli ulcer disease. Ghana Med J 29: 589-594.

29. Ministry of Health, 1999. List of Health Facilities by District, Type and Ownership. Accra, Ghana: Ministry of Health, 1-2.

30. Hayman JA, 1993. Mycobacterium ulcerans infections - the Buruli or Bairnsdale ulcer. Trop Surg 64: 358-360.

31. Ross BC, Marino L, Oppedisano F, Edwards R, Robins-Browne RM, Johnson PD, 1997. Development of a PCR assay for rapid diagnosis of Mycobacterium ulcerans infection. J Clin Microbiol. 35: 1696-1700.

32. Stienstra Y, van der Werf TS, Guarner J, Raghunathan PL, Spotts Whitney EA, van der Graaf WT, Asamoa K, Tappero JW, Ashford DA, King CH, 2003. Analysis of an IS2404-based nested PCR for diagnosis of Buruli ulcer disease in regions of Ghana where the disease is endemic. J Clin Microbiol. 41: 794-797.

33. World Health Organization, 2001. Buruli Ulcer: Diagonosis of Mycobacterium ulcerans. Portaels F, Johnson P, Meyers WM, eds. Geneva: World Health Organization, Geneva, 4-6.

34. Ghana Statistical Services and Macro International Inc., 1998. Ghana Demographic and Health Survey 1998. Calverton, MD: Ghana Statistical Services and Macro International Inc., 14-15.

35. Schlesselman JJ, 1982. Case-Control Studies: Design, Conduct, Analysis. New York: Oxford University Press, 293-324.

36. Armitage P, Berry G, Matthews JNS, 1996. Statistical Methods in Medical Research. Third edition. London: Blackwell Science, 137-144.

37. Revill WDL, Barker DJP, 1972. Seasonal distribution of mycobacterial skin ulcers. Br J Prev Soc Med 26: 23-27. 